\title{
Heidelberg. Die Ophthalmologische Gesellschaft
}

halt am 31. Juli und 1. August d. J. eine außerordentliche Zusammenkunft in Heidelberg ab, bei der in erster Linie Kriegsvorträge gehalten, aber auch andere Themata, so-weit die Zeit reicht, besprochen werden sollen. Für eine Reihe von Kriegs-vorträgen sind bereits Vortragende gewonnen worden.

Der Feldsanitätschef Se. Exzellenz Generalstabsarzt der Armee Dr. von Schjerning hat sich bereit erklärt, wenn es die dienstlichen Verhältnis\&e gestatten, den im Felde stehenden Augenärzten die Teilnahme an der beab-sichtigten Tagung nach Möglichkeit zu erleichtern. Heidelberg.

199

Die wissenschaftlichen Sitzungen finden in dem großen Saale des neuen Kollegiengebäudes (Ludwigsplatz) statt. Ein Epidiaskop steht zur Ver-fügung.

Sonntag, den 30. Juli: Abends 6 Uhr: Vorstandssitzung in der Wohnung von Prof. Wagenmann. Nach 8 Uhr Abends: Zwanglose Zusammenkunft im Stadtgarten.

Montag, den 31. Juli:

Vormittags 9 Uhr: 1. wissenschaftliche Sitzung.

Nachmittags 3 Uhr: 2. wissenschaftliche Sitzung.

Abends 7 1 2 Uhr: Zusammenkunft auf der Molkenkvir. Einfaches Abendessen.

Dienstag, den 1. August:

Vormittags 9 Uhr: 3. wissenschaftliche Sitzung. Nachmittags 3 Uhr: 4. wissenschaftliche Sitzung.

Weitere Vorträge sind unter genauer Angabe des Themas bis spätestens Ende Juni bei dem Schriftführer anzumelden.

Das Manuskript der Vorträge und der Diskussionsbemerkungen, sowie die Vorlagen zu etwaigen A.bbildungen sind noch VOГ Schluß der Zusammenkunft druckfertig an den Schriftführer abzvigeben.

Es wird darauf hingewieEen, daß über die Verhandlungen nichts ver-öffentlicht werden darf, was nicht vorher einer von uns vermittelten Einsicht der Medizinalabteilung des Kriegsministeriums unterzogen worden ist.

Die Mitglieder der Ophthalm. Gesellschaft werden ersucht, etwaige Anderungen ihrer Adresse dem Schriftführer schriftlich mitzuteilen.

Bis jetzt festgesetzte Vorträge:

Augstein-'Brom.herg: Kriegserfahrungen bei Nachtblindheit.

Axenfeld- Freihurg i. Br.: Pathologie und Therapie der Augeninfektionen im Kriege.

Bemheimer-Wien: Über meine Erfahrungen bei Kriegsverletzungen der Opticusbahnen.

Bielschowsky-Marburg: Über Motilitätsstörungen nach Kriegsverletzungen.

Birch-Hirschfeld--Königs, beTg i. Pr.: Hemeralopie im Felde.

Dimmer-Wien: Kriegsverletzungen und sympathische Ophthalmie.

Elschnig-¥$\tau$ a,g; Refraktion und Diensttaviglichkeit.

iZerteZ-Straßburg i. E.: Über Fremdkörperverwundungen des Auges im Kriege. 
v. Hippel-Göttingen: Bedeutung der Stauungspapille bei Hirnschüssen.

Igersheimer-Göttingen: Ein neuer Weg zlur Erkenntnis krankhafter Vor-gänge im Sehnerven.

Krückmann-Berlin: Beurteilung der Militärdiensttauglichkeit.

Krückmann -Berlin: Über Lymph batmen der Retina.

Kuhnt-TioixA: Über plasticche Operationen bei Augenverletzungen im Kriege.

$14 *$

200 Amsterdam.

O/1/8//-Kiel: Über psychogene Kriegsschädigungen.

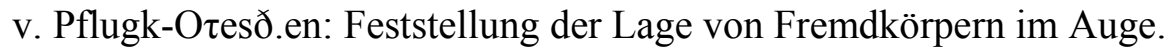

Schieck-Halle a. S.: Die Verhütung der sympathischen Ophthalmie bei Kriegs verletzi ingen.

Stock-Jena: Schießbrillen und andere optische Korrektionen.

v. Szily-Freiburg i. Br.: Ophthalmoskopische Befunde bei Kriegsver-letzungen.

D/8í $\Lambda$ o//-Breslau: Hirnschüsce mit Verletzung der Sehbahnen und Seh-zentren.

Wagenmomn -Heidelberg: Über Scliuß verletzung en des Auges.

Z $\alpha c^{3} / 8-$ Heidelberg: Über Blendung im Fliegerdienst.

Heidelberg, Mai 1916.Prof. Th. Leber-Heidelberg, Prof. A. Wagenmann-Heidelberg,

Vorsitzender d. Vorstandes. Schrif tführer der

Ophthalmologischen Gesellschaft.

Amsterdam. Am 14. A,pril verschied im Alter von 57 Jaliren Prof. Dr. M. Straub, seit 21 Jaliren

Prof essor der Augenheilkunde in Amsterdam. Ein vielseitiger Gelehrter, dem es gelungen ist, für seine Universität eine Muster-Augenklinik zu gründen. Von seiner wissenscliaftlichen

Tätigkeit sind die Üntersuchungen über dieHyalitis und die Theorie der Emmetropie und der Refraktionsanomalien allgemein bekannt. Seine Studien gingen weit über die Grenzen der Augenheilkunde hin, aus: er war mehrere Jahre Heraus-geber der größten Holländischen medizinischen Wochenschrift, verfaßte neben einem Lehrbuche der A.ugenheilkunde in drei Teilen auch ein Lehr-buch der Bakteriologie und vertiefte sieh in manche Fragen der Physiologie und der Psychologie. - · Auch für soziale Fragen hatte er ein offenes Auge: an, der Verbesserung der ärztliclien Ausbildung hat er sein ganzes Leben gearbeitet. Die Refraktionsbestimmungen der Amsterdamer SchuГjugencí, welche ihn einerseits zu seiner Emmetropie-Tlieorie brachten, führten ihn andererseits zu Vorschlägen zur Verbesserung des Unterrichtes in der Volks-schvile. Die heitere Munterkeit, mit welcher er die Hoffnung seiner Kranken rege zu erhalten wußte, wird ihm auch im Auslande, wo er sich oft in Ver-sammlungen und Kongressen zeigte, viele Freunde verschafft haben.

Ein eingehender Nekrolog folgt. Schoute.

U. Urn Einsendung von Separatabdrüeken wird gebeten.

Verantwortücher Redakteur: Prof. Kuhnt in Bonn a. Rh. 